Draft VERSion OCTOBER 26, 2018

Preprint typeset using $\mathrm{LAT}_{\mathrm{EX}}$ style emulateapj v. 14/09/00

\title{
A COLD FRONT IN A PREHEATED GALAXY CLUSTER
}

\author{
John J. Bialek ${ }^{1}$, August E. Evrard ${ }^{1,2} \&$ Joseph J. Mohr ${ }^{3}$ \\ jbialek@umich.edu \\ jmohr@astro.uiuc.edu \\ evrard@umich.edu \\ Draft version October 26, 2018
}

\begin{abstract}
We present a simulated cluster of galaxies, modeled with a pre-heated intracluster medium, that exhibits X-ray features similar to the 'cold fronts' seen in Chandra observations. Mock observations at a particular epoch show factor two discontinuities in X-ray temperature and factor four in surface brightness on a spatial scale $\lesssim 100 \mathrm{kpc}$. Analysis of the cluster's dynamical history reveals that the front is a transient contact discontinuity created by an ongoing merger of two roughly equal mass subgroups. The cold front feature in this realization is amplified by the adiabatic expansion of one of the subgroups following its ablation from the center of its local dark matter potential. The presence of cold front features in a cluster modeled without radiative cooling or magnetic fields implies that such relatively complex physics is not a necessary element of the phenomenon and suggests that the prevalence of such features in high resolution X-ray images of clusters may simply reflect the high frequency of ongoing mergers driven by gravity and comparatively simple hydrodynamics.
\end{abstract}

Subject headings: cosmology: theory - intergalactic medium - methods: numerical - galaxies: clusters: general - instabilities-turbulence-X-rays: galaxies: clusters

\section{INTRODUCTION}

The improved spatial imaging of the hot intracluster medium (ICM) in galaxy clusters by the Chandra Observatory has resulted in the discovery of apparent contact discontinuities, termed "cold fronts", in many clusters (Markevitch et al. 2000; Vikhlinin, Markevitch \& Murray 2001; Mazzotta et al. 2001; Sun et al. 2002). These cold fronts occur at the boundary of a local peak in the X-ray surface brightness and are typically located within a few hundred kpc of the core of the cluster. The fronts exhibit a drop in temperature and corresponding rise in density upon entering the emission peak. The term "cold front" is applied to contrast the phenomenon with a shock front where the temperature would be elevated in the direction of increasing density.

The origin of these cold fronts is thought to be related to cluster mergers (Markevitch et al. 2000). From an extended Press-Schechter treatment, Fujita et al. (2002) predict that up to one-third of present clusters will contain large X-ray subhalos. In this letter, we present evidence supporting the merger origin hypothesis from a simulated cluster evolved under a 'preheated' assumption for ICM evolution (Kaiser 1991; Evrard \& Henry 1991). In this treatment, the proto-ICM gas at high redshift is assumed to lie a fixed, elevated adiabat that results from heating due to star formation and/or AGN activity (Bower 1997; Cavaliere, Menci \& Tozzi 1998; Balogh, Babul \& Patton 1999; Wu, Fabian \& Nulsen 2000; Tozzi \& Norman 2001; Voit \& Bryan 2001). The gas subsequently evolves under gravitationally-driven shock-heating, with magnetic fields and radiative cooling ignored.

As we prepared this work for publication, Nagai \&
Kratsov (2002), using completely independent techniques, present cold front phenomena in a pair of simulations of non-preheated cluster models. Their simulations have higher spatial resolution compared to the one we present, but our Lagrangian simulations have an advantage in allowing the history of gas parcels to be tracked over time. We use this ability to show that material once at the core of a merger progenitor is directly responsible for the cold emission seen at later stages of the merger.

In section 2, we present the simulation and compare its cold front properties to observations. The merger history and thermodynamics responsible for the cold front are examined in section 2.2. Note that all scales quoted in this paper assume a Hubble constant $\mathrm{H}_{0}=70 \mathrm{~km} \mathrm{~s}^{-1} \mathrm{Mpc}^{-1}$.

\section{THE SIMULATED CLUSTER}

Following a study of the effects of preheating on cluster scaling relations (Bialek, Evrard \& Mohr 2001), we have created a new ensemble of 68 simulated clusters that span roughly a decade in final mass. The preheating level of $105.9 \mathrm{keV} \mathrm{cm}^{2}$, favored by the above study, was imposed upon the ensemble. All of the simulations are evolved with the Lagrangian code P3MSPH (Evrard 1988), using the multi-step procedure detailed in Bialek et al. (2001). This ensemble has improved resolution relative to that study; the final hydrodynamic simulation is run on an effective $96^{3}$ particle grid. For the cluster considered in this letter, the gas and dark matter are represented by particles of mass $4.43 \times 10^{8}$ and $3.99 \times 10^{9} M_{\odot}$, respectively. The minimum SPH smoothing length is $40 \mathrm{kpc}$. Twenty outputs, equally spaced in proper time, are stored for each cluster.

The clusters form in a cold dark matter cosmology dominated by vacuum energy density, $\Lambda \mathrm{CDM}$ (Efstathiou et

1 Department of Physics, 1049 Randall Lab, University of Michigan, Ann Arbor, MI 48109

2 Department of Astronomy, Dennison Building, University of Michigan, Ann Arbor, MI 48109

3 Departments of Astronomy and Physics, University of Illinois, 1002 W. Green St, Urbana, IL 61801 
al. 2002). The model assumes a flat spatial geometry with the following parameters: $\Omega_{m}=0.3, \Omega_{\Lambda}=0.7, \Omega_{b}=0.03$, $\sigma_{8}=1.0, \Gamma=0.21$ and $h=0.7$. The Hubble constant is defined as $100 h \mathrm{~km} \mathrm{~s}^{-1} \mathrm{Mpc}^{-1}$; and $\sigma_{8}$ is the power spectrum normalization on $8 h^{-1}$ Mpc scales.
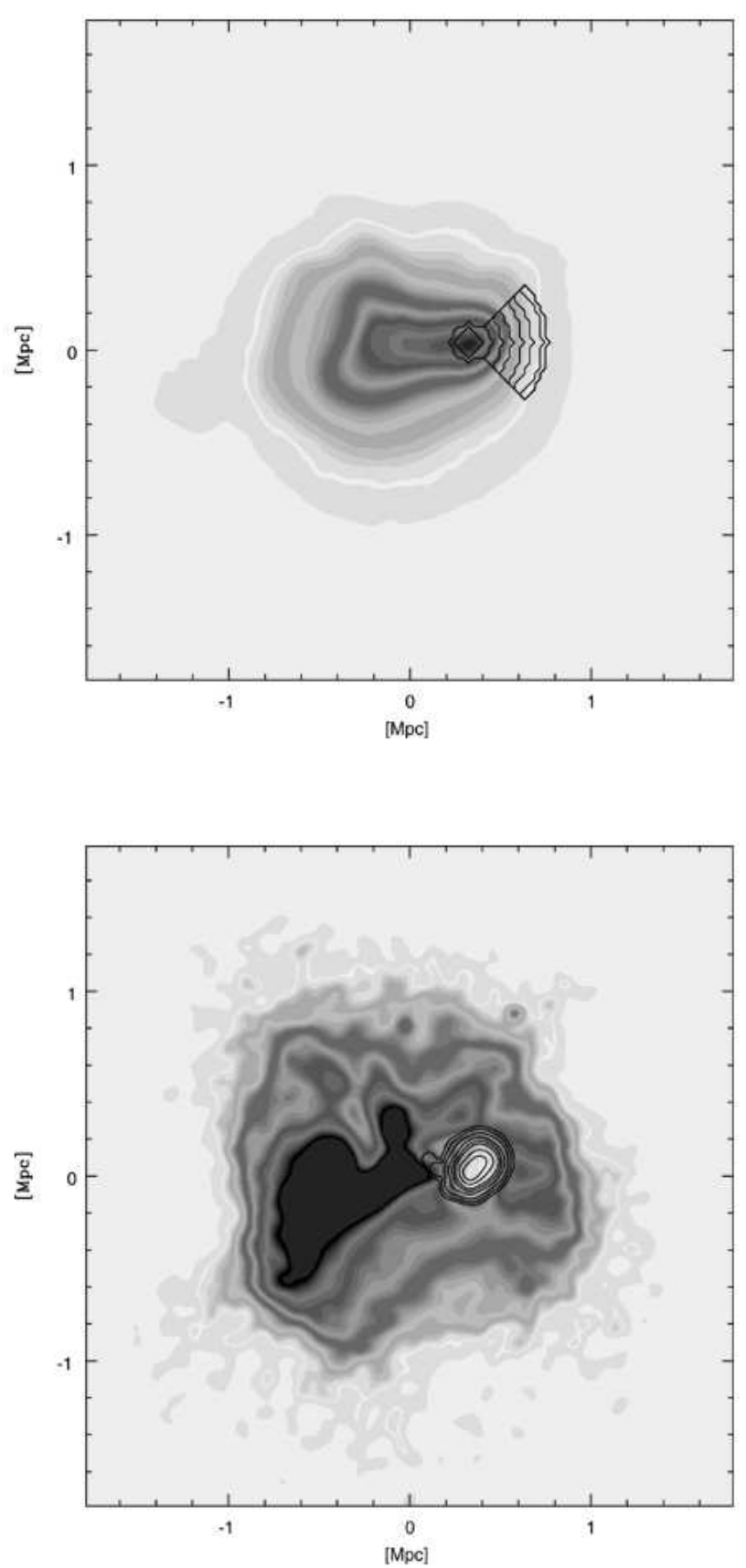

FIG. 1.- (a) Grayscale X-ray surface brightness map (ranging three orders of magnitude) with the general binning structure overlaid (b) Grayscale temperature map $\left(1-3 \times 10^{7} \mathrm{~K}\right)$ with Subgroup 1 contributing surface brightness contours overlaid.

\subsection{A Cold Front Feature}

Examination of some of the mock $\mathrm{X}$-ray surface brightness and temperature maps from the simulation output led to the discovery of a particular cluster with a strong cold front morphology. This cluster, examined at an out- put epoch $z=0.22$, has a mass of $2.86 \times 10^{14} M_{\odot}$ resolved by roughly 35,000 gas and 65,000 dark matter particles, $\mathrm{X}$-ray luminosity of $4.25 \times 10^{43} \mathrm{erg} \mathrm{s}^{-1}$ and an emission weighted temperature of $2.3 \mathrm{keV}$. All values are quoted within the characteristic radius $r_{200}=1.26 \mathrm{Mpc}$ within which the mean interior density is 200 times the critical density at the redshift of examination. The different numbers of gas and dark matter particles reflects the loss of gas within the virial radius caused by preheating (see Fig. 13 of Bialek et al. 2001).

Figure 1 shows $\mathrm{X}$-ray surface brightness and temperature maps of the simulated cluster at $z=0.22$. The morphology of the bright $\mathrm{X}$-ray emission is highly distorted, with a horizontal tongue ending in an off-center surface brightness peak to the right in the figure. The peak in emission is coincident with a cold spot in the temperature map, a signature feature of observed cold fronts.

Following analysis of Abell 2142 by Markevitch et al. (2000), we construct radial profiles within a restricted arc oriented along the steep gradient in the surface brightness (to the right of the cold lump, see Figure 1a). The inner two bins are circular, centered on the surface brightness peak, and extend to $114 \mathrm{kpc}$. The outer six bins are circular arcs in a wedge with outer radius $460 \mathrm{kpc}$ that projects 45 degrees to either side of a horizontal line (parallel to the extended tongue of emission) directed outward from the surface brightness peak. This geometry is used to bin the pixel information in the simulated maps. The resulting temperature profile is shown in Figure 2a. The cool emission peak extends to approximately $230 \mathrm{kpc}$; gas is a factor $1.5-1.9$ cooler inside of the front.

Both the scale of the cold front and the magnitude of the temperature drop are consistent with those reported by many observers. Observed temperature drops range from about a factor 1.5 seen in A1795 (Markevitch, Vikhlinin \& Mazzotta 2001) to about a factor of 3 seen in MS 1455.0+2232 (Mazzotta et al. 2001a). Both A2142 (Markevitch et al. 2000) and A3667 (Vikhlinin, Markevitch \& Murray 2001) are reported to show a factor 2 drop in temperature. Regarding spatial extent, smaller cold fronts are seen, such as MS 1455.0+2232 and A2142 which are both about $70 \mathrm{kpc}$ from the surface brightness peak. Likewise, cold fronts as large as $271 \mathrm{kpc}$ (RX J1720.1+2638, Mazzotta et al. 2001b) and $293 \mathrm{kpc}$ (A3667) are seen. Cold fronts resulting from merger activity would have sizes determined primarily by the masses of the progenitors. From the spectrum of progenitor masses expected from hierarchical clustering (Bower 1991; Lacey \& Cole 1994), one would naturally expect a range of cold front scales to emerge.

For the set of simulation particles identified in the projected wedge used to examine the temperature profile, we derive densities from the normalized emission measure $\int d V \rho^{2} / \int d V \rho$. Figure $2 \mathrm{~b}$ shows a steep drop in density between 200 and $250 \mathrm{kpc}$, the region displaying the factor two rise in temperature. The width of this feature is comparable to the size of the hydrodynamic spatial smoothing at the interior edge of the front (as illustrated by the bar in Figure 2b). The higher resolution models of Nagai \& Kratsov display smaller front widths, but their models do not yet resolve scales $\lesssim 10 \mathrm{kpc}$, the width of the front feature observed by Vikhlinin, Markevitch \& Murray (2001) 


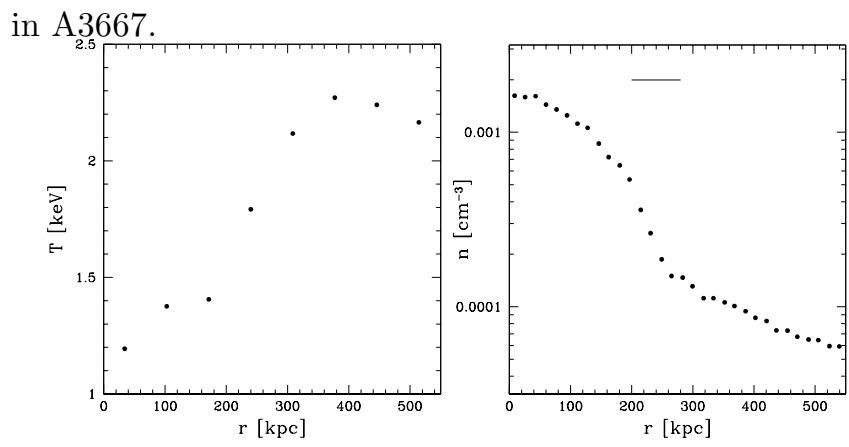

FIG. 2.- (a) Temperature profile across the cold front. (b) The density profile constructed from the emission measure of the particle distribution. The bar in Figure b indicates the average SPH smoothing length in the region of the cold front.

The thermal pressures of particles identified in the projected wedge show no strong discontinuity across the front. The velocity field exhibits some radial compression on the far side of the front, but the associated Mach number is small, $\sim 0.1$. In the next section, we show that the front is a contact discontinuity separating cold, dense material of a subgroup from the surrounding hotter, more tenuous atmosphere of the combined cluster.

\subsection{Cold Front as a Merger Remnant}

Examination of outputs earlier than $z=0.22$ reveals that the cluster is in the throes of a major merger of nearly equal mass progenitors. At a redshift $z=1.07$, a friends-of-friends group finder with linking length 0.15 times the mean gas interparticle separation finds two main subgroups of the cold-front object: Subgroup 1 has a total mass of $8.0 \times 10^{13} M_{\odot}$ and Subgroup 2 has mass $6.4 \times 10^{13} M_{\odot}$. The linking length identifies clumps at densities roughly 500 times the background value.

In order to examine the dynamic and thermodynamic histories of gas within the cores of these subgroups, we employ shorter linking lengths on the same $z=1.07$ output. Values of 0.06 and 0.10 times the mean gas interparticle separation used for Subgroups 1 and 2 result in core gas masses of $3.4 \times 10^{11} M_{\odot}$ and $2.1 \times 10^{11} M_{\odot}$ respectively. The Lagrangian nature of the computation allows the core material in these subgroups to be tracked across all output epochs.

At $z=0.22$, the subgroup cores identified at $z=1.07$ continue to define coherent gas clumps embedded within the larger atmosphere of the merging cluster. Their centroids lie at opposite ends of the tongue of emission shown in Figure 1a. In Figure 1b, we superpose the X-ray emission from the core gas associated with Subgroup 1. This material is responsible for the surface brightness peak and temperature dip at the location of the cold front.

Examination of the orbit of the subgroups shows that they orbit each other with significant angular momentum oriented nearly vertically in the plane of the images shown in Figure 1. The subgroup orbits projected along the rotational axis direction are shown in Figure 3. The location of the most bound gas particle associated with each subgroup is plotted for outputs before and after the viewing redshift $z=0.22$ (indicated by solid symbols). The cores of the subgroups avoid direct collision and the cold front occurs at apogee of their orbit.

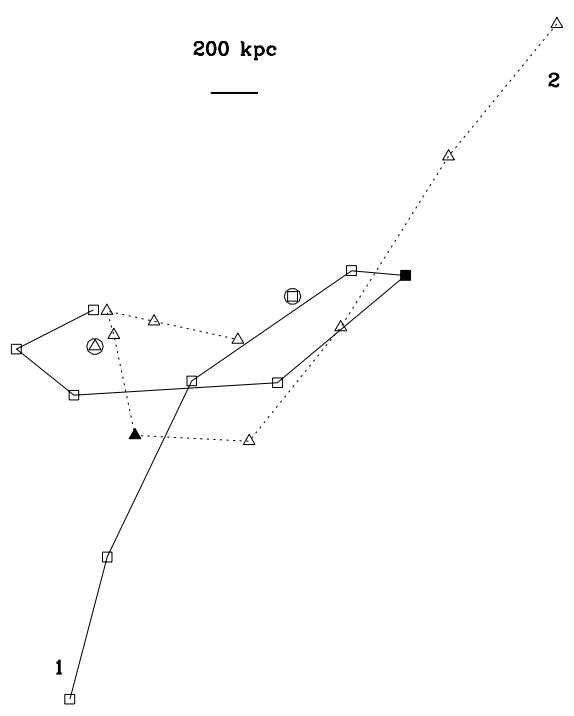

FIG. 3.- Orbits of the subgroup cores in comoving coordinates. The most bound gas particle associated with each subgroup is plotted over 9 outputs ranging from $z=0.54$ to 0 . The earliest epoch is labeled by subgroup number and the $z=0.22$ output, in which the cold front is examined, is indicated by solid symbols. The symbols enclosed by circles indicate the location of the most bound dark matter particle at the viewing epoch. The solid circle indicates the location of the most bound particle for the overall cluster at the viewing epoch. The bar on the top right indicates a $200 \mathrm{kpc}$ scale. The projection used in Figure 1 is for an observer located in the plane of the orbit and viewing the merger from below.

During the encounter, the gas of Subgroup 1 is ablated away from the core of its local confining dark matter potential. At the viewing output, Subgroup 1's gas lags behind its collisionless dark matter component by $300 \mathrm{kpc}$ (see Figure 3). Having been deposited into a shallower part of the potential, the overpressured gas expands adiabatically and cools. The cold nature of the core emission arises from this merger-induced adiabatic expansion.

Subgroup 2, which at as early as $z=0.54$ is somewhat more diffuse than Subgroup 1, experiences different dynamics and does not survive the interaction as comparatively intact. At the viewing output, its gas component has spread out over about a factor two in length and it experiences some increase in entropy due to shock heating.

The different thermodynamic behavior of the two subgroups is shown in Figure 4, where we plot logarithmic changes in density and temperature experienced by the subgroup gas over a time period of $538 \mathrm{Myr}$ (the output time interval of the computation) preceding the viewing epoch $z=0.22$. Just prior to the viewing epoch, particles in Subgroup 1 experience a drop in density and consequentially cool, sliding down the $\gamma=5 / 3$ adiabat shown in the figure as the straight line. The gas of Subgroup 2, which has also been left behind by its dark matter, is in a different state. The leading edge of the gas cloud is becoming more dense as it falls into its dark matter potential well and is gaining entropy as it is being mildly shock heated. This leading edge is leaving behind a diffuse tail of lagging gas, some of which is expanding and cooling adiabatically like the gas of Subgroup 1. 


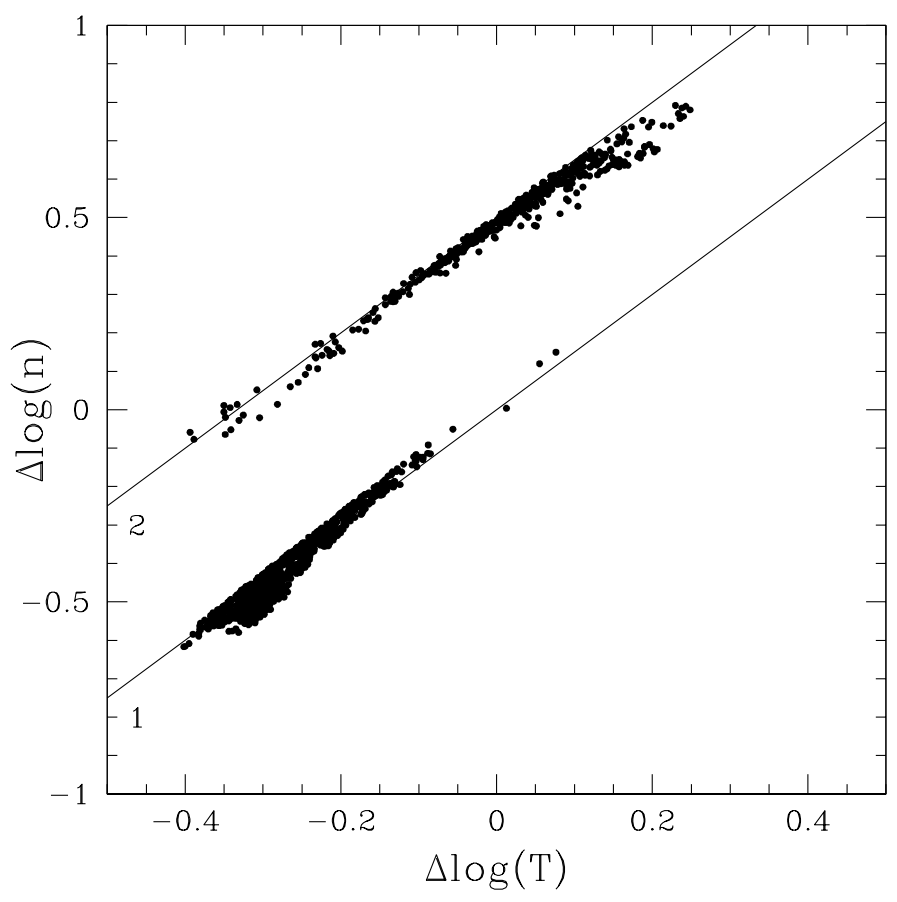

FIG. 4.- Logarithmic changes in density and emperature for particles originating in the cores of Subgroups 1 and 2 (labeled accordingly) over a $538 \mathrm{Myr}$ interval preceding the $z=0.22$ viewing epoch. The superimposed lines marks zero change in entropy. Note that the Subgroup 2 data are offset by 0.5 for clarity.

From analysis of the pair of outputs that precede the viewing epoch, we find that most of the core gas in Subgroup 2 does undergo a period of adiabatic expansion similar to that of Subgroup 1. In the output preceding $z=0.22$, a pair of cold spots appear in the temperature map, but their amplitudes, as well as the amplitudes of their corresponding emission peaks, are somewhat smaller than the features displayed at the viewing epoch of Figure 1. The adiabatic expansion experienced by the core of Subgroup 2 also occurs during the ablation of the gas away from the center of its local gravitational potential. Spatial displacement of cold front gas from the local dark matter peak is also noted in the simulations of Nagai \& Kratsovv (2002). Adiabatic cooling of ablated dense material, coupled with compression and heating of less dense cluster gas in forward of the ablated core, may be the key ingredients involved in the creation of cold fronts by mergers.

Sun et al. (2002) provide observational support for the separation of core gas from its local gravitational poten- tial. They find a scarcity of galaxies within the observed cold front and suggest that the gas is lagging behind its associated galaxies. Assuming that galaxies follow trajectories like those defined by the collisionless dark matter, this conjecture is in agreement with the separation of the gas and dark matter seen in the simulation.

\section{CONCLUSION}

We show that clusters modeled under the assumption of a preheated intracluster medium can exhibit features similar to the cold fronts observed in high resolution spectroscopic imaging of $\mathrm{X}$-ray emission from clusters. We present a particular realization, displaying a temperature dip coincident with a peak in X-ray surface brightness, where the cold front is a transient feature created by ablated core material of a merging subgroup. Freed from its confining local dark matter potential, adiabatic expansion cools the core while its density remains sufficiently high to create a strong feature in emission. This result supports the merger origin assumption for cold fronts and demonstrates that their observed characteristics can be reproduced by a gas dynamic treatment that ignores radiative cooling and magnetohydrodynamics. A similar conclusion using independent methods is reached by Nagai \& Kratsov (2002).

Although these studies provide an existence proof that cold fronts can result from mergers, we do not yet know if all observed cold fronts are consistent with this formation mechanism. It remains to be seen what fraction of mergers result in cold fronts and what combination of parameters - mass ratio, impact parameter, angular momentum, viewing angle — favors such an outcome. Although the cold front in this study occurs when the subcluster's gas strays from its local potential minimum and expands adiabatically, we do not know if this is a necessary condition.

Improved understanding of cold fronts will require more extensive searches within well-defined samples of observed and simulated clusters. The relatively small numbers of observed and simulated cold fronts must be increased to enable secure statistical studies of this phenomenon in the cluster population.

This simulation was produced using the computing facilities at the University of Illinois's National Center for Supercomputing Applications. This work was supported by NASA through grant NAG5-7108 and NSF through grant AST-9803199.

\section{REFERENCES}

Balogh M.L., Babul A., Patton D.R., 1999, MNRAS, 307, 463

Bialek J., Evrard A. \& Mohr J., 2001, ApJ, 555, 597

Bower R.G., 1997, MNRAS, 288, 355

Cavaliere A., Menci N. \& Tozzi P., 1998, ApJ, 501, 493

Efstathiou G. et al. , 2002, MNRAS, 330, L29

Ettore S. \& Fabian A.C., 2001, astro-ph/0110254

Evrard A.E., 1988, MNRAS, 235, 911

Evrard A.E. \& Henry J.P., 1991, ApJ, 383, 95

Fujita Y., Sarazin C.L., Nagashima M. \& Yano T., 2002, ApJ, in press (astro-ph/0205419)

Kaiser N., 1991, ApJ, 383, 104

Markevitch M. et al., 2000, ApJ, 541, 542
Markevitch M., Vikhlinin A. \& Mazzotta P., 2001, ApJ, 562, 153 Mazzotta P, Markevitch M., Forman W.R., Jones C., Vikhlinin A. \& VanSpeybroeck L., 2001a, ApJ, submitted (astro-ph/0108476) Mazzotta P, Markevitch M., Vikhlinin A., Forman W. R., David L.P. \& VanSpeybroeck L., 2001b, ApJ, 555, 205

Nagai D. \& Kravtsov A, 2002, ApJ, submitted (astro-ph/0206469)

Sun M., Murray S., Markevitch M. \& Vikhlinin A., 2002, ApJ, 565, $867 \mathrm{~S}$

Tozzi P. and Norman C., 2001, ApJ, 546, 63

Vikhlinin A., Markevitch M. \& Murray S., 2001, ApJ, 551, 160

Voit G. \& Bryan G., 2001, ApJ, 551, L139

Wu K.K.S., Fabian A.C., Nulsen P.E.J., 2000, MNRAS, 318, 889 